\begin{tabular}{|c|c|}
\hline Title & Majorana Weyl fermions in the chiral superconductor Sr2RuO 4 \\
\hline Author(s) & Nobukane, Hiroyoshi; Tokuno, A kiyuki; Matsuy ama, Toyoki; Tanda, Satoshi \\
\hline Citation & $\begin{array}{l}\text { Physical Review B, 83(14), } 144502 \\
\text { https://doi.org/10.1103/PhysRevB.83.144502 }\end{array}$ \\
\hline Issue Date & 2011-04-01 \\
\hline Doc URL & http:/hdl .handle.net/2115/45276 \\
\hline Rights & @2011 A merican Physical Society \\
\hline Type & article \\
\hline File Information & PRB83-14_144502.pdf \\
\hline
\end{tabular}

Instructions for use 


\title{
Majorana-Weyl fermions in the chiral superconductor $\mathrm{Sr}_{2} \mathrm{RuO}_{4}$
}

\author{
Hiroyoshi Nobukane, ${ }^{1, *}$ Akiyuki Tokuno, ${ }^{1, \dagger}$ Toyoki Matsuyama, ${ }^{2}$ and Satoshi Tanda ${ }^{1}$ \\ ${ }^{1}$ Department of Applied Physics, Hokkaido University, Sapporo 060-8628, Japan \\ ${ }^{2}$ Department of Physics, Nara University of Education, Nara 630-8528, Japan
}

(Received 15 February 2010; revised manuscript received 10 August 2010; published 4 April 2011)

\begin{abstract}
We found Majorana-Weyl fermions in a chiral superconductor $\mathrm{Sr}_{2} \mathrm{RuO}_{4}$. The current-voltage curves reveal anomalous behavior: The induced voltage is an even function of the bias current. The zero-bias conductance peak was observed through the tunnel junction at the edge. The magnetic-field dependence suggests the excitation of the Majorana-Weyl fermions along the closed chiral edge current of the single domain under a bias current. We also discuss the relationship between a change in the chirality and the spontaneous magnetization of the single domain $\mathrm{Sr}_{2} \mathrm{RuO}_{4}$.
\end{abstract}

DOI: 10.1103/PhysRevB.83.144502

PACS number(s): 74.78.Na, 11.30.Er, 73.23.-b, 74.70.Pq

\section{INTRODUCTION}

Parity violation provides important insights about physical phenomena of particle physics and condensed-matter physics, and at least two different parity violation mechanisms are known. The first is due to a quantum anomaly in the Dirac fermions of $(2+1)$-dimensional quantum electrodynamics. ${ }^{1,2}$ Intriguingly, the quantum Hall conductivity in graphene, whose electrical properties are described by Dirac fermions, reveals the parity violation ${ }^{3,4}$ and provides an excellent analog with $(2+1)$-dimensional quantum electrodynamics. Theoretical prediction suggests that the other mechanism is caused by $(2+1)$-dimensional Majorana fermions, where a particle is identified as its own antiparticle. However the parity-violating Majorana fermions have not yet been found. Research on this topic is particularly interesting in relation to both condensed-matter physics and high-energy physics. Moreover, applications to topological quantum computation ${ }^{5-7}$ are expected.

$\mathrm{Sr}_{2} \mathrm{RuO}_{4}{ }^{8}$ is a promising candidate for a spin-triplet chiral $p$-wave superconductor (i.e., electrons are paired with spin $S=1$ and orbital angular momentum $L=1$ ). Since the ground states are twofold degenerate with different chiralities, millimeter-scale $\mathrm{Sr}_{2} \mathrm{RuO}_{4}$ is considered to include chiral domain structures. Recently, the chiral single domain size has been experimentally estimated to be $1 \sim 50 \mu \mathrm{m}^{9-11}$ The millimeter-scale $\mathrm{Sr}_{2} \mathrm{RuO}_{4}$ data should be considered as a result of ensemble averaging over possible chiral domain configurations. Hence transport measurements of samples with a single domain size are very important when studying gapless excitations, ${ }^{12,13}$ the structures and dynamics of unconventional vortices, ${ }^{14,15}$ and quantum Hall effects. ${ }^{16}$ In our previous report, ${ }^{17}$ we suggested that the hydrodynamics of the helical vortex cause the parity violation of current-voltage $(I-V)$ curves. However, this interpretation of the anomalous data has not been confirmed. In this work, we performed transport measurements at the edges of the chiral single domain $\mathrm{Sr}_{2} \mathrm{RuO}_{4}$ because its topological nature appears at the edge.

In this paper, we report anomalous $I-V$ characteristics and magnetic field dependence in a chiral single domain of $\mathrm{Sr}_{2} \mathrm{RuO}_{4}$. The induced voltage $V$ is an even function of the bias current $I$ in the superconducting state. Namely, this violates the parity. The parity-violating $I-V$ curves are dependent on the direction of the applied magnetic field parallel to the $c$ axis.
The results revealed spontaneous magnetization of about 450 Oe and a change in the chirality of the single domain $\mathrm{Sr}_{2} \mathrm{RuO}_{4}$. Indeed the induced voltage $V$ shows the result of the edge conduction. The result of the zero-bias conductance peak in the tunnel junction at an edge of a microscale $\mathrm{Sr}_{2} \mathrm{RuO}_{4}$ single crystal shows the existence of the gapless chiral Majorana state. We suggest that the parity-violating $I-V$ curves with magnetic field dependence result from the low-energy excitation of the right- and the left-handed quasielectrons-holes (Majorana-Weyl fermions) along the closed chiral edge current of the single domain under a bias current.

\section{EXPERIMENT}

To obtain microscale $\mathrm{Sr}_{2} \mathrm{RuO}_{4}$ single crystals, we synthesized $\mathrm{Sr}_{2} \mathrm{RuO}_{4}$ crystals with a solid phase reaction, and then selected microscale $\mathrm{Sr}_{2} \mathrm{RuO}_{4}$ single crystals based on chemical composition and crystallinity results. ${ }^{18}$ We fabricated gold electrodes connected to the sample edges by overlay electron beam lithography. Figure 1(a) shows a micrograph of the measured sample. The size is $13.0 \mu \mathrm{m} \times 6.67 \mu \mathrm{m} \times 0.34 \mu \mathrm{m}$. The electrode spacing is $4.0 \mu \mathrm{m}$. To attach gold electrodes to the sample, we employed a welding technique ${ }^{18,19}$ using electron-beam irradiation as illustrated in Fig. 1(b). Electric transport properties were measured by the four-terminal method using a dilution refrigerator (Kelvinox, Oxford). All the measurement leads were shielded. The lead lines were equipped with low pass RC filters $(R=1 \mathrm{k} \Omega, C=22 \mathrm{nF})$. In the DC measurements, a bias current was supplied by a precise current source (6220, Keithley) and the voltage was measured with a nanovoltmeter (182, Keithley).

\section{RESULTS}

Figure 1(c) shows the superconducting transition temperature $T_{c}=1.59 \mathrm{~K}$ with a transition temperature width $\Delta T \approx 30 \mathrm{mK}$. We measured the temperature dependence of the resistivity for bias current $I= \pm 1 \mu \mathrm{A}$ using a current reversal method. The resistivity is estimated from the size of $4.0 \mu \mathrm{m}$ (length) $\times 5.0 \mu \mathrm{m}$ (width) $\times 0.3 \mu \mathrm{m}$ (thickness), and is obtained by averaging the observed data. From the results, the temperature dependence of the resistivity in the microscale $\mathrm{Sr}_{2} \mathrm{RuO}_{4}$ was consistent with the properties of bulk crystals. ${ }^{20}$ 


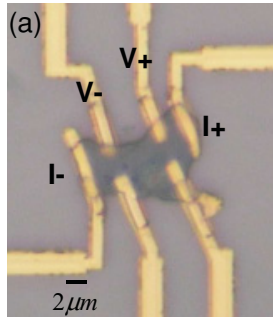

(b)

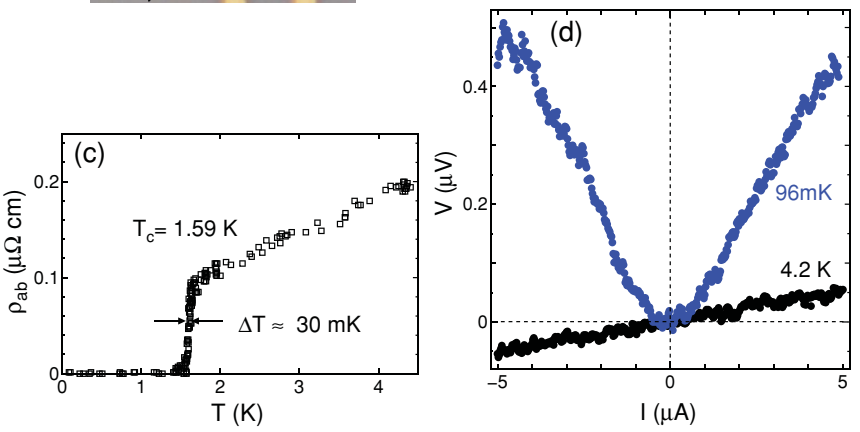

FIG. 1. (Color online) (a) A micrograph of a microscale $\mathrm{Sr}_{2} \mathrm{RuO}_{4}$ single crystal connected to gold electrodes. (b) Schematic of electron beam irradiation welding. (c) Temperature dependence of resistivity of microscale $\mathrm{Sr}_{2} \mathrm{RuO}_{4}$ in zero magnetic field. (d) Voltage $V$ plotted as a function of bias current $I$ at $96 \mathrm{mK}$ and $4.2 \mathrm{~K}$ in zero magnetic field. The induced voltage shows anomalous behavior, which is an even function of the bias current. Notably, the $I-V$ curve at $96 \mathrm{mK}$ implies the parity violation.

We successfully observed the superconducting properties of the microscale $\mathrm{Sr}_{2} \mathrm{RuO}_{4}$ single crystal.

We found the parity-violating $I-V$ characteristics of the microscale $\mathrm{Sr}_{2} \mathrm{RuO}_{4}$ in zero magnetic field below $T_{c}$. Figure 1(d) shows $I-V$ characteristics at $96 \mathrm{mK}$ and $4.2 \mathrm{~K}$. In general, the voltage $V$ in $I-V$ curves is always an odd function of bias current $I$, which is a result of parity conservation. The $I-V$ curve at $4.2 \mathrm{~K}$ is an odd function of $I$. Surprisingly, the $I-V$ curve at $96 \mathrm{mK}$ shows that $V$ has an anomalous component that is an even function of $I$. This implies the parity violation of the $I-V$ characteristics. With decreasing temperature, the anomalous voltage increases in zero magnetic field. The parity violation of $I-V$ curves is observed even in replaced current and voltage leads. The resistance $R$ for $\pm 1 \mu \mathrm{A}$ is zero at $96 \mathrm{mK}$, which is consistent with the result in Fig. 1(c).

The anomalous result seen in the $I-V$ curves is an intrinsic effect of $\mathrm{Sr}_{2} \mathrm{RuO}_{4}$ superconductors. The resistivity of about $0.2 \mu \Omega \cdot \mathrm{cm}$ was observed at $4.2 \mathrm{~K}$ by measuring various electrode arrangements for the six-terminal electrodes in Fig. 1(a). All $I-V$ curves at $4.2 \mathrm{~K}$ exhibited ohmic conduction. Thus the resistivity was unaffected by geometrical factors. The result was confirmed in the AC measurements as reported in Ref. 17. In particular, several samples exhibited the anomalous behavior. We also observed a superconducting gap through the tunnel junction at the edge of the microscale $\mathrm{Sr}_{2} \mathrm{RuO}_{4}$, which was grown in the same batch as the measured sample shown in Fig. 1(a). Our results indicate that the observed anomalous $I-V$ characteristics represent a fundamental superconducting property of microscopic $\mathrm{Sr}_{2} \mathrm{RuO}_{4}$ single crystals.

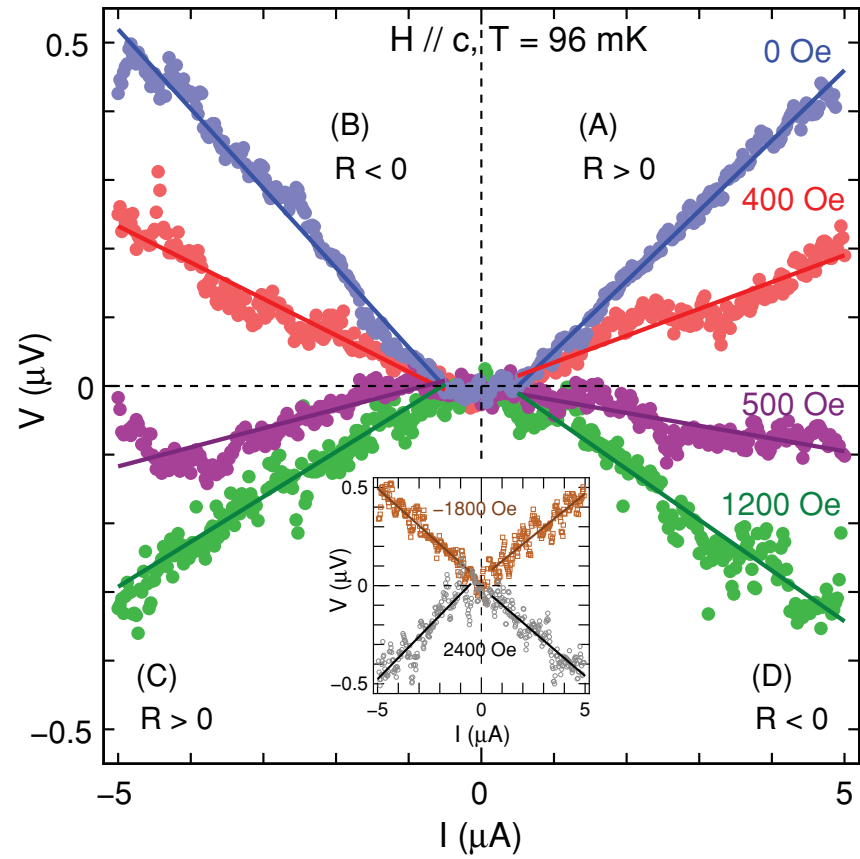

FIG. 2. (Color online) $I-V$ characteristics for several choices of magnetic fields applied parallel to the $c$ axis at $96 \mathrm{mK}$. Applying the magnetic field from zero magnetic field to $1200 \mathrm{Oe}$, the induced voltage changes from a positive voltage of $V(+I)=V(-I)$ to a negative voltage of $-V(+I)=-V(-I)$. The plotted data are fitted to the region between $I=+(-) 0.5 \mu \mathrm{A}$ and $I=+(-) 5.0 \mu \mathrm{A}$ (solid lines). The inset shows $I-V$ characteristics at 2400 and -1800 Oe. We eliminated the offset voltage of about $0.2 \mu \mathrm{V}$, which does not change with the magnetic field.

Figure 2 shows $I-V$ characteristics for several magnetic fields applied parallel to the $c$ axis at $96 \mathrm{mK}$. We found that all of the $I-V$ curves are symmetric with respect to the zero bias current: $V(+I)=V(-I)$ or $-V(+I)=-V(-I)$. Applying the magnetic field from zero magnetic field to $1200 \mathrm{Oe}$, the resistance $R$ on a bias current $I>0$ changes from a positive value $(R>0)$ to a negative value $(R<0)$. On the other hand, for a bias current $I<0$, a negative $R$ changes to a positive $R$. The inset shows $I-V$ curves under higher magnetic fields. The $I-V$ curve for magnetic field $H=500$ Oe shows zero resistance for a low-bias current. The zero resistance seen in that case may indicate superconductivity.

To analyze the result in Fig. 2, we plotted the magnetic-field dependence of conductance for a bias current $I>0$ or $I<0$ as shown in Fig. 3. Here the conductance was extracted from the slope of the linear $I-V$ characteristics in Fig. 2. The conductance of conventional superconductors with a magnetic field has symmetric curves with respect to zero magnetic field and has an infinite conductance at zero magnetic field. Figure 3 , however, shows that the sample has a finite conductance at the zero magnetic field, and the point at which the conductance diverges shifts to a finite $H \approx 450$ Oe. Furthermore, the sign of the conductance is reversed around the critical magnetic field 450 Oe. In contrast, the conductance is robust against a magnetic field applied in the opposite direction $(H<0)$. In a magnetic field away from $450 \mathrm{Oe}$, the conductance 


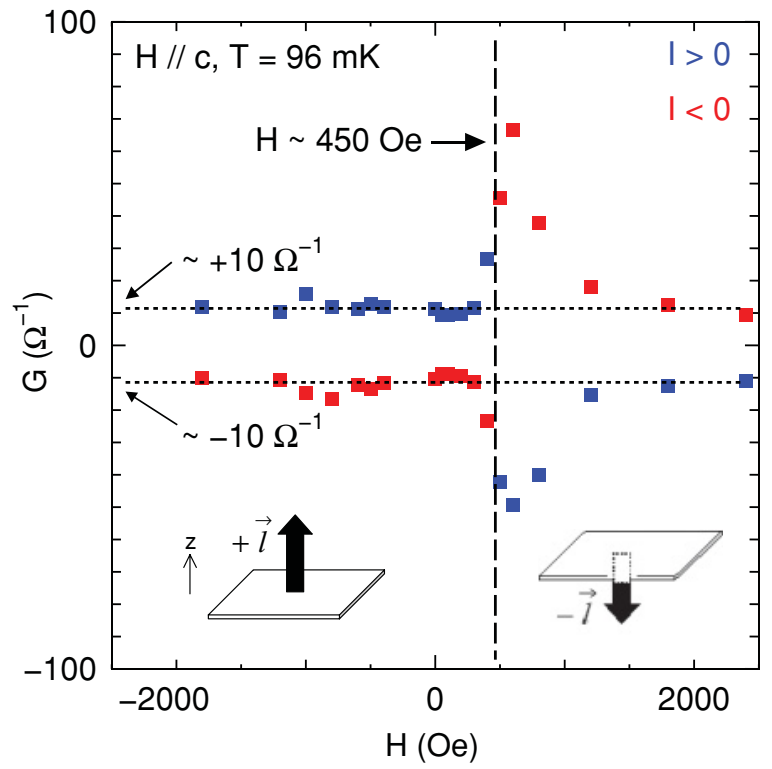

FIG. 3. (Color online) Magnetic field dependence of conductance $G$ on bias current. The shift of the infinite conductance point to $H \sim 450$ Oe and its change of its sign are shown. The conductance of $\pm 10 \Omega^{-1}$ is represented by horizontal dotted lines. The insets show the $\pm \vec{l}$ chiral domains in the $a b$ plane. Here we assume that a $+\vec{l}$ chiral single domain with broken time-reversal symmetry is spontaneously formed in the $+z$ direction in zero magnetic field.

asymptotically reaches $\pm 10 \Omega^{-1}$ as shown by the horizontal dotted lines in Fig. 3.

Figure 4(a) shows the differential conductance $d I / d V$ as a function of the bias voltage for magnetic fields applied parallel to the $c$ axis at $60 \mathrm{mK}$ through the tunnel junction
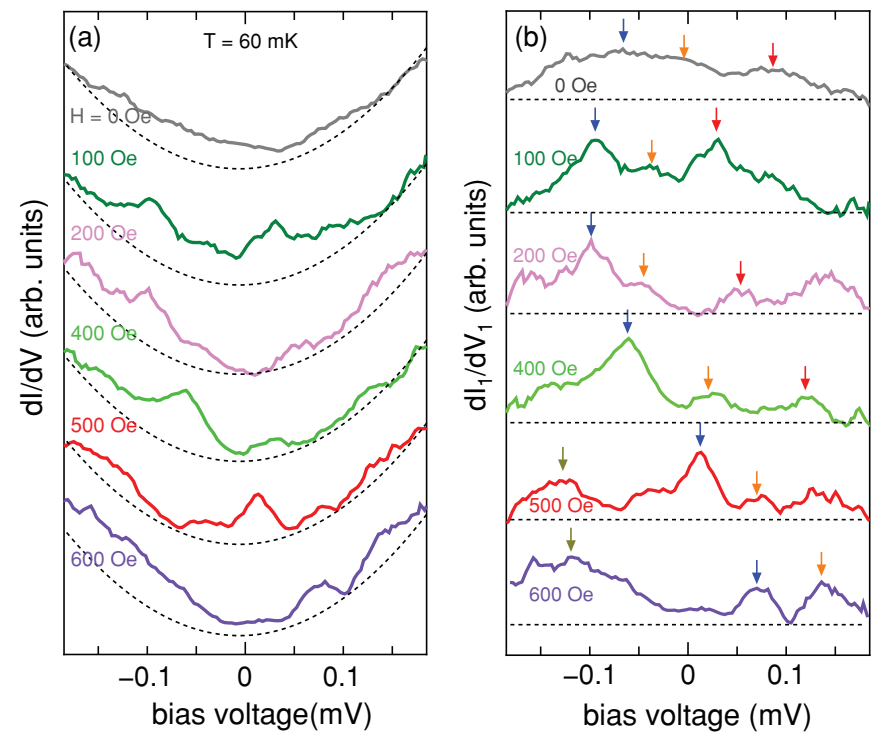

FIG. 4. (Color online) (a) Differential conductance $d I / d V$ versus bias voltage for magnetic fields applied parallel to the $c$ axis at $60 \mathrm{mK}$. The dotted line represents the result of the fitting curves. The conductance peaks in the vicinity of zero bias voltage were observed. (b) Magnetic field dependence of the extracted spectra with conductance peaks. The peak positions are represented by the arrows. at the edge of the microscale $\mathrm{Sr}_{2} \mathrm{RuO}_{4}$. The tunneling spectra were measured by a standard lock-in technique. The junction resistance exhibited $19.3 \mathrm{k} \Omega$ in zero magnetic field at $60 \mathrm{mK}$. This is comparable with a quantum resistance of $\sim h / e^{2}$, where $e$ is the charge of an electrons and $h$ is Planck's constant. We observed differential conductance peaks in the vicinity of zero-bias voltage. To analyze the peaks, we subtract background part of the spectra from the curves in Fig. 4(a). The results of the extracted spectra $\left(d I_{1} / d V_{1}\right.$ curves) are shown in Fig. 4(b). We found that the position of the conductance peak for the applied magnetic field was shifted from a minus bias voltage to a plus voltage as shown by the blue arrows in Fig. 4(b). Under an external magnetic field of 500 Oe, the zero-bias conductance peak was observed. These results were similar to the behavior of the anomalous $I-V$ curves with magnetic field dependence in Fig. 2.

\section{DISCUSSION}

To understand the physical origin of the parity-violating $I-V$ characteristics, we first discuss the dependence of the chirality of the single domain of $\mathrm{Sr}_{2} \mathrm{RuO}_{4}$ on an external magnetic field. The chiral domain can explain the shift in the singular point of the conductance and the change of its sign in Fig. 3. Now let us suppose that a chiral vector of a single domain, $+\vec{l}$, spontaneously points in the $+z$ direction ( $c$ axis) in zero magnetic field as shown in the left panel of the inset in Fig. 3. The vector $\vec{l}$ represents the pair angular momentum parallel to the $c$ axis. Assuming that a magnetic field $H_{\mathrm{ex}}$ applied in the $-z$ direction (opposite to $+\vec{l}$ ) would suppress the chirality of the domain, the chiral vector of the domain would eventually point in the opposite direction (i.e., $-\vec{l}$ ) in a positive effective magnetic field $H_{\text {eff }}>0$, where the effective magnetic field is defined as $H_{\mathrm{eff}}=\left(H_{\mathrm{ex}}-450 \mathrm{Oe}\right)$. In contrast, if the assumption is correct, the magnetic field applied in the $+z$ direction is expected to affect the change of the chiral domain with $+\vec{l}$, and the sign of the conductance does not change in $H_{\text {eff }}<0$. In fact, according to Fig. 3, the assumption appears to be valid. Our sample size $\sim 10 \mu \mathrm{m}$ is comparable to the single domain size $1 \sim 50 \mu \mathrm{m}$ estimated from the experiments, ${ }^{9-11}$ so the sample should exhibit a single domain structure. Thus the microscale $\mathrm{Sr}_{2} \mathrm{RuO}_{4}$ itself is a chiral single domain with spontaneous magnetization and the applied magnetic field causes the chirality of the domain to change.

How can we interpret the anomalous $I-V$ curves in terms of the peculiar transport phenomena in the chiral single domain? We focus on the existence of Majorana-Weyl fermions in the edge of $\mathrm{Sr}_{2} \mathrm{RuO}_{4}$. The edge modes of spin-polarized chiral superconductors are fermionic Bogoliubov quasiparticles, which are mixture of electrons and holes. The absolute distinction is blurred in order to modify their interaction with the surrounding sea of Cooper pairs. ${ }^{21}$ Thus, they should not be treated as ordinary fermions, but as Majorana fermions. ${ }^{22,23}$ With a spontaneous magnetic field of $450 \mathrm{Oe}$ as shown in Fig. 3, $\mathrm{Sr}_{2} \mathrm{RuO}_{4}$ may realize spin-polarized Cooper pairs. Both chiral edge supercurrent and quasielectrons-holes current flow in the edge. The Majorana-Weyl fermions pass through a gapless state and generate the induced voltage. In Fig. 4, the conductance peaks with magnetic field dependence in the vicinity of zero-bias voltage show the existence of the gapless 
(b)Right-handed quasiholes
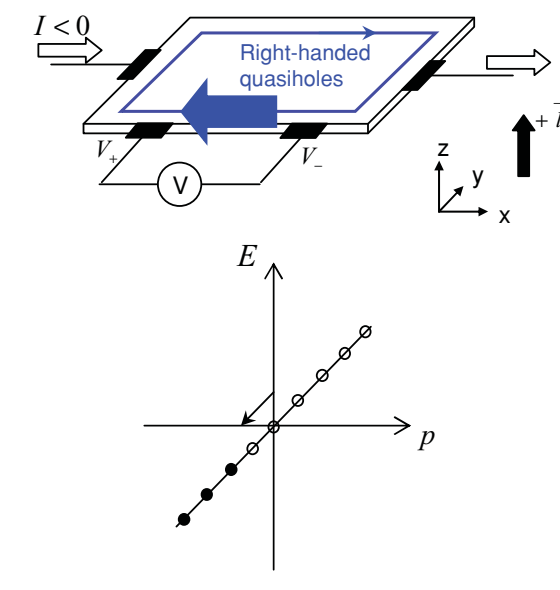

(c)Left-handed quasiholes
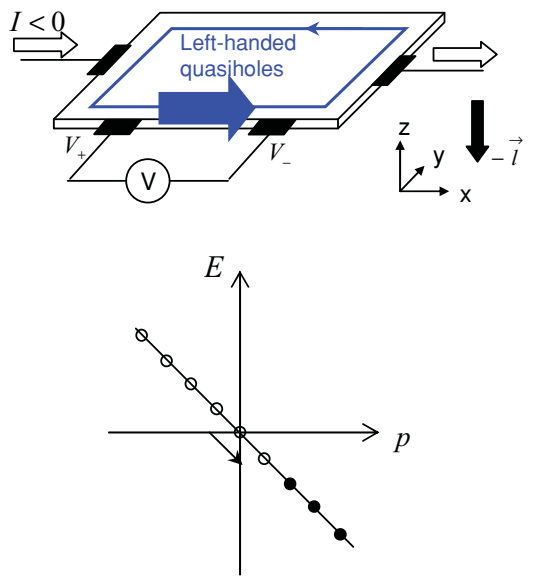

(a)Right-handed quasielectrons
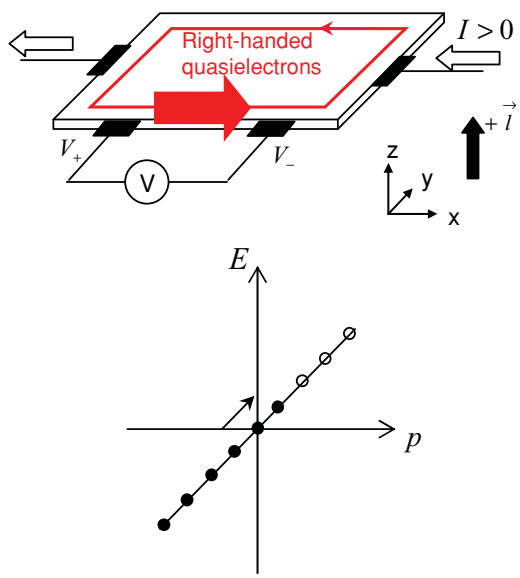

(d) Left-handed quasielectrons
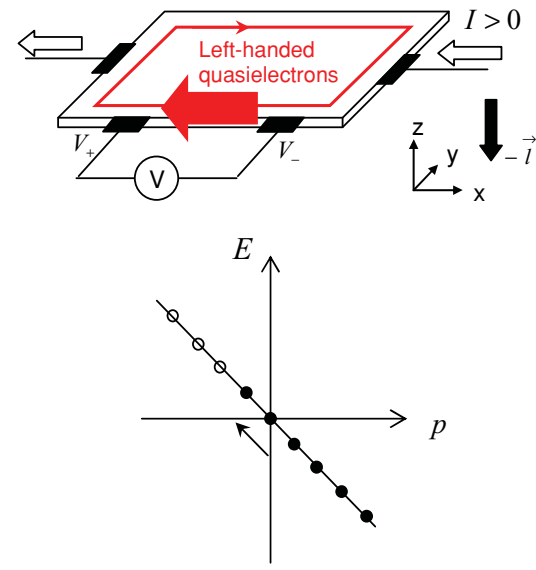

FIG. 5. (Color online) A circulating model of Majorana-Weyl fermions along the closed edge of the sample and energy spectrum in chiral edge states. Under a bias current $I>0(I<0)$, right- and left-handed quasielectrons (quasiholes) are created by the excitation from the Fermi surface (point). The closed edge of the single domain is regarded as a one-dimensional annular line. Here we assume a clockwise direction for the $+\vec{l}$ domain as the positive momentum $p$. The solid and open circles represent quasielectrons and quasiholes, respectively. The arrangement of panels (a)-(d) corresponds to regions (A)-(D) in Fig. 2.

chiral Majorana state at the edge. The transport phenomena at least in a low-energy state appear to be governed by the edge excitation of the Majorana-Weyl fermions. Based on the result of the peak shift in Fig. 4(b), we think that the applied magnetic field affects the topological conduction path in the chiral edge excitation. It is possible to explain the $I-V$ curves in terms of the characteristics of Majorana-Weyl fermions.

The parity-violating $I-V$ curves can be understood as being the result of the selective excitation of Majorana-Weyl fermions along a closed edge of the single domain in the bias current direction (i.e., $I>0$ or $I<0$ ). As shown in Fig. 5, we assume that each region of the $I-V$ curve can be related to the paths of quasielectrons or quasiholes carrying the current: Panels (a)-(d) in Fig. 5 respectively correspond to regions (A)-(D) shown in Fig. 2. Here we assume that the chiral edge supercurrent of the $+\vec{l}$ domain flows clockwise in the $+z$ direction along the boundary even in the absence of the bias current. With a bias current $I>0$, when right-handed quasielectrons are created by low-energy excitation from the Fermi surface (point), they circulate along the closed line [Fig. 5(a)]. By contrast, for a bias current $I<0$, the right-handed quasiholes created by the excitation circulate in the opposite direction [Fig. 5(b)]. As a consequence, an anomalous induced voltage of $V(+I)=V(-I)$ was observed. Furthermore, similarly to the above explanation, when the $-\vec{l}$ domain, which has a chiral edge supercurrent that flows counterclockwise, produces left-handed quasielectrons and quasiholes, a voltage of $-V(+I)=-V(-I)$ is induced as shown in Fig. 5(c) and 5(d). The excitation of quasiholes to the negative energy is considered as the same excitation of quasielectrons to the positive energy in Majorana fermions. ${ }^{23}$ Since, in an effective magnetic field $H_{\text {eff }} \approx 0$, the number of spin-polarized Cooper pairs decreases, no voltage is induced. The excitation is determined by the bias current and the 
direction of the applied magnetic field. In this manner, the parity-violating $I-V$ curves can be understood as relating to the excitation of the Majorana-Weyl fermions. This is evidence for the existence of parity-violating Majorana-Weyl fermions in the chiral single domain of $\mathrm{Sr}_{2} \mathrm{RuO}_{4}$.

Let us discuss the resistivity in both the normal $(T \geqslant$ $T_{c}$ ) and the superconducting states $\left(T \ll T_{c}\right)$. As shown in Fig. 1(c), the normal resistivity $\rho_{N}\left(=R_{N} \frac{w t}{L}\right)$ is $0.10 \mu \Omega \mathrm{cm}$ at temperature just above $T_{c}$ with a sample width $w \approx 5 \mu \mathrm{m}$, a electrode spacing $L \approx 4 \mu \mathrm{m}$ and a thickness $t \approx 0.3 \mu \mathrm{m}$. If we assume the width of the state of the edge excitations to be $\lambda_{a b}$, we can estimate the edge resistivity at $96 \mathrm{mK}$ to be $\rho_{\text {edge }}=0.11 \mu \Omega \mathrm{cm}\left(=R^{\star} \frac{\lambda_{a b} t}{L}\right)$ using the constant resistance $R^{\star}=0.1 \Omega$ in Fig. 3, and a magnetic penetration length $\lambda_{a b} \approx 152 \mathrm{~nm}$. The edge resistivity $\rho_{\text {edge }}$ is equivalent to the normal resistivity $\rho_{N}$ at temperature just above $T_{c}$. Namely, the conduction path in the superconducting state becomes the edge. Thus our result reflects the quasielectrons-quasiholes of the edge current. From these results such as the parity-violating $I-V$ curve and the edge conduction, we can conclude that the anomaly of the induced voltage is the signature of the observation of the Majorana-Weyl fermions carrying the chiral current along the closed edge of the single domain $\mathrm{Sr}_{2} \mathrm{RuO}_{4}$ under a bias current.

We mainly describe the contribution of Majorana-Weyl fermions based on the edge transport properties. At present, it seems to be more reasonable than considering an inhomogeneous current path because the simple conduction path appears topologically at the edge in the gapless chiral state. In general, however, the experimental data for $\mathrm{Sr}_{2} \mathrm{RuO}_{4}$ include not only the contribution of Majorana-Weyl fermions, but also that of other exotic effects such as unconventional vortices. We believe that further studies will reveal these contributions in detail from measurements of the electric properties in various electrode arrangements that involve Hall-bar geometry.

Finally we discuss the physical implications of our results. As is well known, the Majorana-Weyl fermions may describe the nature of neutrinos in high-energy physics. Thus, the Majorana-Weyl fermions of chiral single domain $\mathrm{Sr}_{2} \mathrm{RuO}_{4}$ might be regarded as neutrinos in condensed-matter systems. This means a possibility of studying cosmology in the laboratory. Our results suggest a novel effective action like the Chern-Simons term in $(2+1)$-dimensional quantum electrodynamics, where it is hard to find the most relevant action of the theory without a phenomenon. In addition to attracting purely scientific interest, the anomalous transport phenomena have the potential applications as regards realizing the topological quantum computation of non-Abelian statistics.

\section{SUMMARY}

In summary, we have observed Majorana-Weyl fermions in the edge of a chiral single domain of $\mathrm{Sr}_{2} \mathrm{RuO}_{4}$ through electric transport. In four terminal measurements, the $I-V$ curves exhibited parity violation with magnetic field dependence. We observed the zero-bias conductance peak in the tunnel junction at the edge. The $\mathrm{Sr}_{2} \mathrm{RuO}_{4}$ itself provides spontaneous magnetization. An applied magnetic field changes the chirality of the single domain. We show that the MajoranaWeyl fermions are excited along the chiral edge current under a bias current and are detected in the edge of $\mathrm{Sr}_{2} \mathrm{RuO}_{4}$.

\section{ACKNOWLEDGMENTS}

We thank K. Inagaki, Y. Asano, K. Ichimura, K. Yamaya, S. Takayanagi, K. Konno, T. Tsuneta, S. Tsuchiya, J. Ishioka, I. Kawasaki, K. Tenya, H. Amitsuka, and T. Honma for experimental help and fruitful discussions. We also are grateful to A. J. Niemi, E. Babaev, M. Chernodub, J. Goryo, and N. Hatakenaka. This work was supported by a Grant-in-Aid for the 21st Century COE program on "Topological Science and Technology" from the MEXT. H.N. was supported by KAKENHI (No. 21840004).
*Present address: Department of Physics, Hokkaido University, Sapporo 060-0810, Japan.

${ }^{\dagger}$ Present address: DPMC-MaNEP, University of Geneva, 24 quai Ernest-Ansermet, 1211 Geneva 4, Switzerland.

${ }^{1}$ G. W. Semenoff, Phys. Rev. Lett. 53, 2449 (1984).

${ }^{2}$ T. Matsuyama, Prog. Theor. Phys. 77, 711 (1987).

${ }^{3}$ K. S. Novoselov, A. K. Geim, S. V. Morozov, D. Jiang, M. I. Katsnelson, I. V. Griorieva, S. V. Dubonos, and A. A. Firsov, Nature (London) 438, 197 (2005).

${ }^{4}$ Y. Zhang, Y.-W. Tan, H. L. Stormer, and P. Kim, Nature (London) 438, 201 (2005).

${ }^{5}$ C. Nayak, S. H. Simon, A. Stern, M. Freedman, and S. D. Sarma, Rev. Mod. Phys. 80, 1083 (2008).

${ }^{6}$ M. Dolve, M. Heiblum, V. Umansky, A. Stern, and D. Mahalu, Nature (London) 452, 829 (2008).

${ }^{7}$ I. P. Radu, J. B. Miller, C. M. Marcus, M. A. Kastner, L. N. Preiffer, and K. W. West, Science 320, 899 (2008).

${ }^{8}$ A. P. Mackenzie and Y. Maeno, Rev. Mod. Phys. 75, 657 (2003).

${ }^{9}$ J. Xia, Y. Maeno, P. T. Beyersdorf, M. M. Fejer, and A. Kapitulnik, Phys. Rev. Lett. 97, 167002 (2006).
${ }^{10}$ F. Kidwingira, J. D. Strand, D. J. V. Harlingen, and Y. Maeno, Science 314, 1267 (2006).

${ }^{11}$ H. Kambara, S. Kashiwaya, H. Yaguchi, Y. Asano, Y. Tanaka, and Y. Maeno, Phys. Rev. Lett. 101, 267003 (2008).

${ }^{12}$ G. E. Volovik, Pis'ma Zh. Eksp. Teor. Fiz. 61, 935 (1995) [JETP Lett. 61, 958 (1995)].

${ }^{13}$ G. E. Volovik, Pis'ma Zh. Eksp. Teor. Fiz. 66, 492 (1997) , [JETP Lett. 66, 522 (1997)].

${ }^{14}$ E. Babaev, Phys. Rev. Lett. 88, 177002 (2002).

${ }^{15}$ S. B. Chung, H. Bluhm, and E.-A. Kim, Phys. Rev. Lett. 99, 197002 (2007).

${ }^{16}$ J. Goryo and K. Ishikawa, Phys. Lett. A 260, 294 (1999).

${ }^{17}$ H. Nobukane, K. Inagaki, K. Ichimura, K. Yamaya, S. Takayanagi, I. Kawasaki, K. Tenya, H. Amitsuka, K. Konno, Y. Asano, and S. Tanda, Solid State Commun. 149, 1212 (2009).

${ }^{18}$ H. Nobukane, K. Inagaki, S. Tsuchiya, Y. Asano, K. Ichimura, K. Yamaya, S. Takayanagi, I. Kawasaki, K. Tenya, H. Amitsuka, and S. Tanda, Jpn. J. Appl. Phys. 49, 020209 (2010). 
${ }^{19}$ K. Inagaki, T. Toshima, S. Tanda, and K. Yamaya, Appl. Phys. Lett. 86, 073101 (2005).

${ }^{20}$ A. P. Mackenzie, R. K. W. Haselwimmer, A. W. Tyler, G. G. Lonzarich, Y. Mori, S. Nishizaki, and Y. Maeno, Phys. Rev. Lett. 80, 161 (1998).
${ }^{21}$ F. Wilczek, Nature Phys. 5, 614 (2009).

${ }^{22}$ N. Read and D. Green, Phys. Rev. B 61, 10267 (2000).

${ }^{23}$ X.-L. Qi, T. L. Hughes, S. Raghu, and S.-C. Zhang, Phys. Rev. Lett. 102, 187001 (2009). 